\title{
INTERVENTION EFFECT OF SUPPORTIVE GROUP THERAPY AND PHYSICAL EXERCISE ON THE QUALITY OF LIFE OF CAMGER PATIENTS
}

\author{
Dimitra Papastergiou, , A, B, D Dimitrios Kokaridas, , , A, D Konstantinos Bonotis, , A, D \\ Nikolaos Digelidis, ${ }^{2, A, D}$ Asterios Patsiaouras ${ }^{2, C, D}$ \\ ${ }^{1}$ Faculty of Medicine, School of Health Science, University of Thessaly, Greece \\ ${ }^{2}$ Department of Physical Education \& Sport Science, University of Thessaly, Greece \\ A Study Design; ${ }^{\mathrm{B}}$ Data Collection; ${ }^{\mathrm{C}}$ Statistical Analysis; ${ }^{\mathrm{D}}$ Manuscript Preparation \\ Address for correspondence: \\ D. Papastergiou \\ Aisxylou 1, 41336 Larisa, Greece \\ E-mail: d_papastergiou@yahoo.gr
}

\begin{abstract}
Ahstract Psychological distress is a multidimensional concern affecting patients' ability to cope with cancer, its physical symptoms, and treatments.

This study examined the effect of an exercise program and a group psychotherapy program on the quality of life of Greek cancer patients.

The sample consisted of 39 cancer patients (10 males and 29 females), assigned randomly in three groups of 13 patients each group, that is, a control, an exercise and a psychotherapy group. The duration of the training program for the individuals of the exercise group was 10 weeks at a frequency of two sessions per week, 60 minutes each session. The patients of the psychotherapy group received a 10 weeks' supportive-expressive group therapy, at a frequency of one time per week, of 90 minutes each meeting. The quality of life SF-36 questionnaire was administered to examine the short-term effect of both programs prior and after intervention on quality of life. Control group individuals did not participate in any program and they just filled in the SF-36 questionnaire prior and after intervention.

Improvement in "vitality" $(p=0.006)$ and mental health subscale $(p=0.011)$ was statistically significant between pre and post measures in the supportive therapy group. All other domains exhibit no significant changes. In the exercise group, physical functioning, role functioning and emotional role values were also improved but not to the point to generate statistically significant results. The findings of the present study support the positive impact of psychotherapy intervention on vitality and mental health component of patients with cancer, followed in less extent by the beneficial effect of the exercise program.
\end{abstract}

Key worlds quality of life, cancer survivors, exercise, group psychotherapy

\section{Introduction}

Cancer is a chronic disease threatening the life and physical integrity of the patient that extends over time and raises issues of psychological significance for the individual. As such, cancer diagnosis is a psycho-traumatic event, while treatment and progression of the disease compose a complex and extremely stressful experience for the 
patient that alleviates over time and affects both in short- and long- term the overall functionality level of sufferers (Trudel-Fitzgerald et al., 2017).

Emerged clinical phenomena that characterize the development of post-traumatic syndrome include constant disease engaging, intrusive thoughts of death and refusal or avoidance of anything that recalls the disease including available therapeutic options. Furthermore, relapses of the disease may be even more traumatic than its initial attack and consequent arising anxiety psychologically disrupts patient's quality of life (Stanton, Bower, 2015).

Quality of life is a subjective and multidimensional concept and health-related quality of life concerns the subjective assessment of disease incidence and its treatment at the physical, psychological and social level of functionality and well-being. Therapeutic interventions that are often ambulatory and adjuvant therapies such as chemotherapy and radiotherapy, affect patients at a physical, psychological, social and mental level, resulting in anxiety, depression and quality of life reduction (Naughton, Weaver, 2014).

Psychological distress is a multidimensional concern affecting patients' ability to cope with cancer, its physical symptoms and treatment consequences (Gundelach, Henry, 2016) and if untreated, may lead to lowered quality of life, reduced coping and compatibility with the disease and even suicide (Klaassen et al., 2017). Appropriate interaction with cancer patients is necessary for dealing with side effects of cancer therapy and improving their quality of life (QoL) (Badger et al., 2013).

Psychological interventions exert beneficial effects on patients' mood and further on their quality of life. Yeh et al in their systematic review reported that these interventions had a positive effect that improved psychological distress and several QoL domains (Yeh, Chung, Hsu, Hsu, 2014) and supported a potential bio-behavioral pathway relevant to cancer survivorship (Wenzel et al., 2015). Furthermore, exercise interventions are associated with reduced depression, pain and sense of fatigue, leading cancer survivors to the development of stress coping mechanisms, vitality enhancement and quality of life improvements even for patients who receive chemotherapy (Craft, Vaniterson, Helenowski, Rademaker, Courneya, 2012; Gerritsen, Vincent, 2015).

Nevertheless, psychological outcomes in research efforts including both psychological and exercise interventions in their design are yet to be drawn. This study examined the effect of a supportive group therapy program and an exercise program on the quality of life of Greek cancer patients.

\section{Methods}

This study and its randomized control trial protocol and procedures were reviewed and approved by the DPESS University of Thessaly bioethics committee.

\section{Sample}

The sample consisted of 39 patients ( 10 males and 29 females, with mean age of $57.8 \pm 15.98$ and $58.3 \pm 9.05$ respectively) with cancer, any type (19 with breast cancer, 7 with colorectal cancer, 3 with ovarian cancer and 6 with other types of respiratory, hematological and other malignancies), regardless of previous kind of treatment (surgery alone, and/or adjunct therapy - radiation/chemotherapy) as diagnosed by their oncologist. All patients were registered members of the Larissa cancer patients association, they volunteered to participate in the study and they were all able to keep on normal activity with minor symptoms of the disease according to the Karnofsky performance scale (Azevedo, Viamonte, Castro, 2013). 
Pre-intervention phase included a meeting prior initiation of the program with information provided by the researcher regarding benefits, content and safety of exercise and group psychotherapy intervention procedures. Thus, all patients were aware of any possible risks associated with their participation and they agreed to sign the consent form of participation in the study, to complete the SF-12 instrument prior and after intervention and to have their information used for research purposes. Next, the sample was assigned randomly into three groups (a control group, an exercise group and a psychotherapy group, of 13 patients each one).

\section{Procedure}

\section{Psychotherapy (SEGT) group}

Supportive-expressive group therapy (SEGT) is a non-structured intensive form of group psychotherapy that provides several psychosocial advantages, applied mainly to women with metastatic breast cancer and other cancer conditions too. SEGT orientation is existential and cognitive, encouraging communication, emotional expression, sharing of mutual beneficial ideas, development of coping strategies and network support of cancer patients so as to address their arising concerns caused by the disease (Butler et al., 2009).

Studies suggest that SEGT implementation affects positively indicators such as physical symptoms, depression and anxiety, mental discomfort, emotional suppression, quality of life, and survival rate of patients, although few contradictory results has also been reported in research studies (Azevedo et al., 2013; Butler et al., 2009). Engaging in SEGT provides bonding opportunities among patients and creates a setting for self-disclosure that reduces fear and confusion, thus, SEGT has a special feature designed for application to cancer patients (Butler et al., 2009; Hill, Amir, Muers, Connolly, Round, 2003).

In this study, a 10 weeks' supportive-expressive group therapy (SEGT) was applied to patients of the psychotherapy group at a frequency of one session (meeting) per week of 90 minutes each session, with the researcher and a specialized psychologist present. In each meeting, SEGT intervention was designed to use emotions as a marker for what may be important to focus so as to encourage communication and deal with isolation feelings, changes in self and body image, reordering of life priorities and fear concerns of dying and death. No drop outs occurred during sessions.

\section{Exercise group}

Individuals of the exercise group received a 10 weeks training program, at a frequency of two (2) training sessions each week, of 60 minutes per session. Since this sample of patients had heterogeneous types of the disease and there are no precise guidelines for exercise prescription for each different type of cancer (Azevedo et al., 2013), this study followed general exercise recommendations that include duration of 20 to 60 minutes per session at a moderate exercise intensity recommended for cancer patients, described as the physical effort that makes participants breathe somewhat harder than normal but does not interfere with conversation (Clark et al., 2007). Furthermore, the Borg Scale (Borg, 1982) was used a subjective mean of exercise intensity, with 12 and 14 (from "fairly light" to "somewhat hard") specified as the desired perceived exertion in each session. Training frequency was 2 times per week that is better tolerated for de-conditioned populations (Schmitz et al., 2010) as compared to training frequency of 3-5 sessions per week suggested for healthy participants.

Each session included a 5-minute warm-up period of flexibility and respiratory exercises followed by 10 minutes of walking, 35 minutes of balance, co-ordination and aerobic activities and a 10 minutes cool-down 
period of breathing and relaxation. The exercise intervention was supervised and group-based with modifications as needed according to individualized needs and performance, leading all participants to successfully complete the exercise program. A swimming session was also included following patients' request. Finally, a medical clearance was provided by their physician prior the start of the program to ascertain that no general contraindications to exercise participation were present (Moreno-Smith, Lutgendorf, Sood, 2010).

\section{Control Group}

Control group individuals did not participate in any exercise or supportive therapy program or and they just filled in the SF 36 questionnaire prior and after intervention.

\section{Instruments}

The Greek version (Pappa, Kontodimopoulos, Niakas, 2005) of the SF-36 Health Survey (Ware, Sherbourne, 1992) was used to assess health-related quality of life. Good psychometric properties of the SF-36 were previously documented in cancer patients (Bunevicius, 2017; Treanor, Donnelly, 2015) and in cancer survivors (Reulen et al., 2006). The SF-36 examines individual perceptions regarding quality of life in relation to eight different items of functioning that is, physical functioning, bodily pain, role limitations due to physical problems and general health that are summarized into a physical component score, and social functioning, energy/vitality, role limitations due to emotional problems and mental health that constitute a mental component score. Scores range from 0 (worse) to 100 (best) possible range with higher scores indicating a better health related quality of life.

\section{Statistical Analysis}

The Statistical Package of Social Sciences (SPSS 22.0) was used for statistical analysis. Normality of sample distribution was examined using Shapiro-Wilk analysis (Ghasemi, Zahediasl, 2012). Since sample distribution was normal, parametric tests (paired sample t test, ANOVA) were applied to examine differences between groups in pre and post measures. No missing data or data transformations were noted. Statistical significance was set at $p=0.05$.

\section{Results}

A normal test distribution was noted using Shapiro-Wilk analysis (Reulen et al., 2006), since no significant results were observed for all variables and criterion value was high. An increase of vitality score was statistically significant $(p=0.006)$ in post measures for the psychotherapy (SEGT) group while mental health component was also improved at a statistically significant level $(p=0.011)$. In the exercise group, post intervention values in physical functioning, role functioning and emotional role were improved but not to the extent to produce statistically significant results.

No such differences were noted in control group either (Table1). 
Table 1. Pre and post-intervention SF-36 scores

\begin{tabular}{|c|c|c|c|}
\hline Variables & Group (pre-post-test) & Mean \pm SD & $p$ \\
\hline 1 & 2 & 3 & 4 \\
\hline \multirow{6}{*}{ Physical Functioning } & SEGT (pre) & $63.64 \pm 27.26$ & \multirow{2}{*}{0.397} \\
\hline & SEGT (post) & $58.18 \pm 23.69$ & \\
\hline & Exercise (pre) & $75.42 \pm 17.90$ & \multirow{2}{*}{0.736} \\
\hline & Exercise (post) & $77.08 \pm 18.02$ & \\
\hline & Control (pre) & $61.36 \pm 27.39$ & \multirow{2}{*}{0.541} \\
\hline & Control (post) & $59.09 \pm 30.32$ & \\
\hline \multirow{6}{*}{ Role Functioning } & SEGT (pre) & $65.38 \pm 36.14$ & \multirow{2}{*}{0.721} \\
\hline & SEGT (post) & $61.54 \pm 39.02$ & \\
\hline & Exercise (pre) & $68.75 \pm 44.11$ & \multirow{2}{*}{0.909} \\
\hline & Exercise (post) & $70.96 \pm 35.01$ & \\
\hline & Control (pre) & $38.46 \pm 41.60$ & \multirow{2}{*}{1,000} \\
\hline & Control (post) & $38.46 \pm 45.20$ & \\
\hline \multirow{6}{*}{ Role Emotional } & SEGT (pre) & $78.79 \pm 37.34$ & \multirow{2}{*}{0.258} \\
\hline & SEGT (post) & $60.61 \pm 41.68$ & \\
\hline & Exercise (pre) & $63.89 \pm 45.97$ & \multirow{2}{*}{0.748} \\
\hline & Exercise (post) & $69.44 \pm 41.34$ & \\
\hline & Control (pre) & $58.33 \pm 40.51$ & \multirow{2}{*}{0.137} \\
\hline & Control (post) & $44.44 \pm 35.77$ & \\
\hline \multirow{6}{*}{ Vitality } & SEGT (pre) & $59.23 \pm 21.20$ & \multirow{2}{*}{0.006} \\
\hline & SEGT (post) & $77.31 \pm 9.92$ & \\
\hline & Exercise (pre) & $65.83 \pm 15.93$ & \multirow{2}{*}{0.940} \\
\hline & Exercise (post) & $65.42 \pm 17.77$ & \\
\hline & Control (pre) & $61.67 \pm 26.31$ & \multirow{2}{*}{0.570} \\
\hline & Control (post) & $63.33 \pm 27.33$ & \\
\hline \multirow{6}{*}{ Mental Health } & SEGT (pre) & $65.09 \pm 27.37$ & \multirow{2}{*}{0.011} \\
\hline & SEGT (post) & $89.64 \pm 9.42$ & \\
\hline & Exercise (pre) & $70.55 \pm 21.04$ & 0251 \\
\hline & Exercise (post) & $59.27 \pm 28.39$ & 0.201 \\
\hline & Control (pre) & $74.33 \pm 26.50$ & 0891 \\
\hline & Control (post) & $74.00 \pm 25.67$ & 0.091 \\
\hline \multirow{6}{*}{ General Health } & SEGT (pre) & $63.46 \pm 17.49$ & \multirow{2}{*}{0.531} \\
\hline & SEGT (post) & $59.62 \pm 23.05$ & \\
\hline & Exercise (pre) & $71.11 \pm 18.33$ & \multirow{2}{*}{0.938} \\
\hline & Exercise (post) & $71.67 \pm 13.92$ & \\
\hline & Control (pre) & $57.73 \pm 26.21$ & \multirow{2}{*}{0.821} \\
\hline & Control (post) & $58.18 \pm 25.72$ & \\
\hline \multirow{6}{*}{ Bodily Pain } & SEGT (pre) & $70.00 \pm 23.10$ & \multirow{2}{*}{0.229} \\
\hline & SEGT (post) & $60.91 \pm 28.09$ & \\
\hline & Exercise (pre) & $80.23 \pm 20.26$ & \multirow{2}{*}{0.641} \\
\hline & Exercise (post) & $76.82 \pm 18.68$ & \\
\hline & Control (pre) & $67.71 \pm 32.64$ & 0.406 \\
\hline & Control (post) & $69.38 \pm 32.14$ & 0.400 \\
\hline
\end{tabular}




\begin{tabular}{|c|c|c|c|}
\hline 1 & 2 & 3 & 4 \\
\hline \multirow{6}{*}{ Social Functioning } & SEGT (pre) & $76.04 \pm 21.62$ & \multirow{2}{*}{0.339} \\
\hline & SEGT (post) & $66.67 \pm 26.83$ & \\
\hline & Exercise (pre) & $72.92 \pm 27.09$ & \multirow{2}{*}{0.845} \\
\hline & Exercise (post) & $75.00 \pm 26.11$ & \\
\hline & Control (pre) & $70.83 \pm 33.43$ & \multirow{2}{*}{0.615} \\
\hline & Control (post) & $72.92 \pm 30.07$ & \\
\hline \multirow{6}{*}{ Physical Health Subscale } & SEGT (pre) & $264.06 \pm 88.16$ & \multirow{2}{*}{0.239} \\
\hline & SEGT (post) & $236.56 \pm 98.71$ & \\
\hline & Exercise (pre) & $302.19 \pm 81.71$ & \multirow{2}{*}{0.906} \\
\hline & Exercise (post) & $298.94 \pm 36.53$ & \\
\hline & Control (pre) & $222.22 \pm 128.53$ & \multirow{2}{*}{0.171} \\
\hline & Control (post) & $228.89 \pm 128.04$ & \\
\hline \multirow{6}{*}{ Mental Health Subscale } & SEGT (pre) & $271.91 \pm 101.32$ & \multirow{2}{*}{0.400} \\
\hline & SEGT (post) & $243.85 \pm 92.32$ & \\
\hline & Exercise (pre) & $278.65 \pm 93.17$ & \multirow{2}{*}{0.685} \\
\hline & Exercise (post) & $265.03 \pm 102.40$ & \\
\hline & Control (pre) & $247.43 \pm 120.91$ & \multirow{2}{*}{0.543} \\
\hline & Control (post) & $237.20 \pm 106.84$ & \\
\hline
\end{tabular}

\section{Discussion}

In this study, findings support the positive effect of SEGT implementation on vitality and mental health of cancer patients, referring to less sense of fatigue and improved energy levels, less anxiety and depression symptoms and recovery of emotional control, leading to an improved psychological wellbeing and quality of life perception. As for exercise, scores in all SF-36 domains did not change substantially compared to SEGT application, although individuals of the experiment group improved their post scores in relation to less limitations experienced due to physical or mental health difficulties caused by the disease. Nevertheless, pre-post differences were not statistically significant.

Comparisons with previous intervention studies for cancer patients including exercise in their design are challenging due to differences in their methodological design. J.K. Gerittsen and A.J. Vincent (2015) in their systematic review and meta-analysis of randomized control trials (RCTs) concerning exercise and quality of life of cancer patients, showed that only 6 out of 16 of these researches had a duration of less or equal to 10 weeks that is comparable to ours, with 3 of them producing statistically significant results. Two of these studies included mixed cancer patients population like our study, receiving, however, either a high-intensity cardiovascular and heavy resistance training combined with relaxation and body awareness training of 9 hours weekly for 6 weeks (Andersen et al., 2013) or a combined walking and strength training program for five days per week for six weeks (Samuel et al., 2012) compared to the medium intensity exercise program twice per week of our study. Of the three aforementioned studies, only S.R. Samuel et al. (2012) used the SF-36 questionnaire along with 3 other studies out of the 16 RCTs included in the systematic review of J.K. Gerittsen and A.J. Vincent (2015).

Overall, most studies reported a significant improvement in terms of fatigue. Nevertheless, although exercise especially when supervised exerts beneficial effects on fatigue and depression with improvements in the relevant quality of life fields among cancer survivors (Gerritsen, Vincent, 2015), beyond these areas, exercise contribution 
to total quality of life improvement needs further investigation in short-term interventions (Monga et al., 2007; Hejazi, Bahrami, Keshvari, Alavi, 2017). The most recent meta-analysis of L.M. Buffart et al. (2017) concluded that exercise effects on QOL were significant, but small in general. According to L.M. Buffart et al. (2017) exercise, especially when supervised, improves quality of life and physical function of cancer patients with different clinical or demographic characteristics during and following treatment, thus, there is consistent evidence to support exercise implementation as part of cancer care. Nevertheless, issues such as patient's motivation to participate, the duration of the program and the subjective evaluation of quality of life (Korstjens et al., 2008) require further investigation. In our sample of participants with different cancer types, all patients were in the after treatment period where only intense aerobic exercise such as cycling or rowing for at least 12 months in certain cancer groups has been reported to have a significant effect (Gerritsen, Vincent, 2015).

On the other hand, psychological interventions are less time consuming and demand minimal physical effort from cancer patients. In this regard, patients of any age could attend intervention sessions and some early results might be obvious within a couple of weeks. Even a 3-week communicational program consisting of distributing educational booklets, practices, and phone follow-ups may have positive impact on psychological distress (Hejazi et al., 2017). The randomized clinical trial of L. Wenzel et al. (2015) in 204 survivors of cervical cancer examining the effect of psychosocial telephone counseling on QOL domains and its association with biomarkers confirmed mood and quality of life benefits related to positive bio-indicator changes, supporting in this way a potential bio-behavioral pathway relevant to cancer survivorship.

A. Torre-Luque et al.,(2016) in their meta-analysis noted that psychological treatments promote greater QoL for survivors, with a noteworthy effect on reducing somatic anxiety, sleep problems or other symptomatology as a consequence of medical treatments, although these benefits tend to reduce in patients under active treatment. As patients recover from cancer they have to adapt to different daily conditions than before. In this transition phase, the physical and psychological consequences could be efficiently managed within the context of psychological support. Nevertheless, psychological interventions effect size is rather small regarding improvement of QOL and reduction in depressive symptomatology, thus, strategies towards these goals should be included in future psychological treatments (Knobf, Thompson, Fennie, Erdos, 2014).

Unique survivor characteristics should always be considered when recommending different interventions that might improve psychological QOL (Badger et al., 2013), such as the exercise and SEGT interventions used in our study, to further recommend appropriate care for cancer patients. Variables related to the individual's pre-morbid psychological characteristics and the manner in which this individual copes with the cancer are more related to QOL scores than to cancer-related variables such as treatment types and cancer severity (Brunault et al., 2016). Future studies should consider optimizing such interventions, by taking into account patients' unique needs.

Overall, this study underlines the effect of a SEGT program on improving mental health and vitality of cancer patients leading to an improved quality of life perception. The small sample size, the mixed population of cancer patients and the relatively short duration of intervention all constitute limitations. In this context, it seems that for the cancer patients of our study sharing their experience with others through a SEGT approach was more effective to improve their QOL perceptions compared to individual efforts to cope with the disease through physical effort. Exercise seemed to have a small but not significant effect in post mental health scores but only psychotherapy enhanced QOL domains of cancer patients. Future researches with larger samples and longer periods of intervention 
in selected patients (e.g. specific age groups, non-mixed population) are needed to further verify QOL gains derived from exercise and/or SEGT strategies.

\section{Implications for Practice}

The results of this study support the importance of supportive-expressive group therapy (SEGT) as an effective intervention tool that improve vitality and mental health of cancer patients leading to an enhanced quality of life perception. Furthermore, it provides in detail the design of psychotherapy and exercise procedures that could be applied in everyday nursing practice or adopted by future studies. Finally, it recommends that exercise should not be overlooked as a potential mean to improve psychological wellbeing of cancer patients, creating the need for future everyday practices and research efforts to combine psychotherapy and exercise interventions so as to maximize psychological benefits.

\section{References}

Andersen, C., Rørth, M., Ejlertsen, B., Stage, M., Møller, T., Midtgaard, J., Quist, M., Bloomquist, K., Adamsen, L. (2013). The effects of a six-week supervised multimodal exercise intervention during chemotherapy on cancer-related fatigue. Eur $\mathrm{J}$ Oncol Nurs, 17, 331-339.

Azevedo, M.J., Viamonte, S., Castro, A. (2013). Exercise prescription in oncology patients: General principles. Rehabilitación, 47, 170-178.

Badger, T.A., Segrin, C., Figueredo, A.J., Harrington, J., Sheppard, K., Passalacqua, S., Pasvogel, A., Bishop, M. (2013). Who benefits from a psychosocial counselling versus educational intervention to improve psychological quality of life in prostate cancer survivors? Psychol Health, 28, 336-354.

Borg, G.A. (1982). Psychophysical bases of perceived exertion. Med Sci Sports Exerc, 14, 377-381.

Brunault, P., Champagne, A.L., Huguet, G., Suzanne, I., Senon, J.L., Body, G., Camus, V. (2016). Major depressive disorder, personality disorders, and coping strategies are independent risk factors for lower quality of life in non-metastatic breast cancer patients. Psycho-Oncology, 25 (5), 513-520.

Buffart, L.M., Kalter, J., Sweegers, M.G., Courneya, K.S., Newton, R.U., Aaronson, N.K., Jacobsen, P.B., May, A.M., Galvão, D.A., Chinapaw, M.J., Brug, J. (2017). Effects and moderators of exercise on quality of life and physical function in patients with cancer: An individual patient data meta-analysis of 34 RCTs. Cancer Treat Rev, 52, 91-104.

Bunevicius, A. (2017). Reliability and validity of the SF-36 Health Survey Questionnaire in patients with brain tumors: a cross-sectional study. Health and quality of life outcomes, 15, 92.

Butler, L.D., Koopman, C., Neri, E., Giese-Davis, J., Palesh, O., Thorne-Yocam, K.A., Dimiceli, S., Chen, X.H., Fobair, P., Kraemer, H.C., Spiegel, D. (2009). Effects of supportive-expressive group therapy on pain in women with metastatic breast cancer. Health Psychology, 28, 579.

Clark, M.M., Vickers, K.S., Hathaway, J.C., Smith, M., Looker, S.A., Petersen, L.R., Pinto, B.M., Rummans, T.A., Loprinzi, C.L. (2007). Physical activity in patients with advanced-stage cancer actively receiving chemotherapy. Journal of Supportive Oncology, 5 , 487-493.

Craft, L.L., Vaniterson, E.H., Helenowski, I.B., Rademaker, A.W., Courneya, K.S. (2012). Exercise effects on depressive symptoms in cancer survivors: a systematic review and meta-analysis. Cancer Epidemiology and Prevention Biomarkers Prev, 21, 3-19.

Gerritsen, J.K., Vincent, A.J. (2015). Exercise improves quality of life in patients with cancer: a systematic review and meta-analysis of randomized controlled trials. British Journal of Sports Medicine, 50, 796-803.

Ghasemi, A., Zahediasl, S. (2012). Normality tests for statistical analysis: a guide for non-statisticians. International Journal of Endocrinology and Metabolism, 10, 486-489.

Gundelach, A., Henry, B. (2016). Cancer-Related Psychological Distress: A Concept Analysis. Clinical Journal of Oncology Nursing, 20, 630-634.

Hejazi, F., Bahrami, M., Keshvari, M., Alavi, M. (2017). The Effect of a Communicational Program on Psychological Distress in the Elderly Suffering from Cancer. Iran J Nurs Midwifery Res, 22, 201-207. 
Hill, K.M., Amir, Z., Muers, M.F., Connolly, C.K., Round, C.E. (2003). Do newly diagnosed lung cancer patients feel their concerns are being met? European Journal of Cancer Care, 12, 35-45.

Klaassen, Z., Arora, K., Wilson, S.N., King, S.A., Madi, R., Neal, D.E. Jr, Kurdyak, P., Kulkarni, G.S., Lewis, R.W., Terris, M.K. (2017). Decreasing suicide risk among patients with prostate cancer: Implications for depression, erectile dysfunction, and suicidal ideation screening. Urol Oncol, S1078-1439, 30470-30472.

Knobf, M.T., Thompson, A.S., Fennie, K., Erdos, D. (2014). The effect of a community-based exercise intervention on symptoms and quality of life. Cancer Nurs, 37, E43-50.

Korstjens, I., May, A.M., van Weert, E., Mesters, I., Tan, F., Ros, W.J., Hoekstra-Weebers, J.E., van der Schans, C.P., van den Borne, B. (2008). Quality of life after self-management cancer rehabilitation: a randomized controlled trial comparing physical and cognitive-behavioral training versus physical training. Psychosom Med, 70, 422-429.

Monga, U., Garber, S.L., Thornby, J., Vallbona, C., Kerrigan, A.J., Monga, T.N., Zimmermann, K.P. (2007). Exercise prevents fatigue and improves quality of life in prostate cancer patients undergoing radiotherapy. Arch Phys Med Rehabil, 88, 1416-1422.

Moreno-Smith, M., Lutgendorf, S.K., Sood, A.K. (2010). Impact of stress on cancer metastasis. Future Oncology, 6, $1863-1881$.

Naughton, M.J., Weaver, K.E. (2014). Physical and mental health among cancer survivors: considerations for long-term care and quality of life. N C Med J, 75, 283-286.

Pappa, E., Kontodimopoulos, N., Niakas, D. (2005). Validating and norming of the Greek SF-36 Health Survey. Qual Life Res, 14, 1433-1438.

Reulen, R.C., Zeegers, M.P., Jenkinson, C., Lancashire, E.R., Winter, D.L., Jenney, M.E., Hawkins, M.M. (2006). The use of the SF-36 questionnaire in adult survivors of childhood cancer: evaluation of data quality, score reliability, and scaling assumptions. Health Qual Life Outcomes, 4, 77.

Samuel, S.R., Arun, M.G., Babu, A.S., Vidyasagar, M.S. (2012). Effect of exercise training on functional capacity \& quality of life in head \& neck cancer patients receiving chemoradiotherapy. Indian J Med Res, 137, 515-520.

Schmitz, K.H., Courneya, K.S., Matthews, C., Demark-Wahnefried, W., Galvão, D.A., Pinto, B.M., Irwin, M.L., Wolin, K.Y., Segal, R.J., Lucia, A., Schneider, C.M., von Gruenigen, V.E., Schwartz, A.L. (2010). American College of Sports Medicine roundtable on exercise guidelines for cancer survivors. Med. Sci. Sports Exerc, 42, 1409-1426.

Stanton, A.L., Bower, J.E. (2015). Psychological Adjustment in Breast Cancer Survivors. Adv Exp Med Biol, 862, $231-242$.

Torre-Luque, A., Gambara, H., López, E., Cruzado, J.A. (2016). Psychological treatments to improve quality of life in cancer contexts: A meta-analysis. International Journal of Clinical and Health Psychology, 16, 211-219.

Treanor, C., Donnelly, M. (2015). A methodological review of the Short Form Health Survey 36 (SF-36) and its derivatives among breast cancer survivors. Qual Life Res, 24, 339-362.

Trudel-Fitzgerald, C., Tworoger, S.S., Poole, E.M., Zhang, X., Giovannucci, E.L., Meyerhardt, J.A., Kubzansky, L.D. (2017). Psychological Symptoms and Subsequent Healthy Lifestyle after a Colorectal Cancer Diagnosis. Health Psychol.

Ware, J.E., Sherbourne, C.D. (1992). The MOS 36-item short-form health survey (SF-36): I. conceptual framework and item selection. Medical Care, 30, 473-483.

Yeh, M.L., Chung, Y.C., Hsu, M.Y., Hsu, C.C. (2014). Quantifying psychological distress among cancer patients in interventions and scales: a systematic review. Curr Pain Headache Rep, 18, 399.

Wenzel, L., Osann, K., Hsieh, S., Tucker, J.A., Monk, B.J., Nelson, E.L. (2015). Psychosocial telephone counseling for survivors of cervical cancer: results of a randomized biobehavioral trial. J Clin Oncol, 33, 1171-1179.

Cite this article aS: Papastergiou, D., Kokaridas, D., Bonotis, K., Digelidis, N., Patsiaouras, A. (2019). Intervention Effect of Supportive Group Therapy and Physical Exercise on the Quality of Life of Cancer Patients. Central European Journal of Sport Sciences and Medicine, 1 (25), 5-13. DOI: 10.18276/cej.2019.1-01. 